\title{
Construction of Polymeric Metal-Organic Nanocapsule Networks via Supramolecular Coordination-Driven Self-Assembly
}

\author{
Li Shao ${ }^{\S}$, Bin Hua*†, Xiangquan $\mathrm{Hu}^{\S}$, David Stalla ${ }^{\#}$, Steven P. Kelley ${ }^{\S}$, and Jerry L. Atwood* ${ }^{\S}$ \\ ${ }^{\S}$ Department of Chemistry, University of Missouri, Columbia, Missouri 65211, United States \\ \# Electron Microscopy Core Facility, University of Missouri, Columbia, Missouri 65211, United States \\ 'Department of Chemistry, Zhejiang University, Hangzhou 310027, P. R. China \\ Email: huabin@zju.edu.cn;Atwoodj@missouri.edu
}

\section{Supporting Information (14 pages)}

1. Materials and methods $\quad$ S2

2. The crystal structure of $\mathbf{M O N C - 1}$ S6

3. TEM and STEM images of PEG400@MONCs and PEG8000@MONCs S6

4. ${ }^{1} H$ NMR spectra of MONC-1,EG@MONC,PEG400@MONCs and PEG8000@MONCs

5. FTIR spectra of EG@MONC,PEG400@MONCs and PEG8000@MONCs

6. TGA spectra of MONC-1, EG@MONC,PEG400@MONCs and PEG8000@MONCs S9

7. Optical microphotographs of MONC-1, and EG@MONC S9

8. Photographs of the solution formed from $\mathrm{Mg}\left(\mathrm{NO}_{3}\right)_{2} \cdot 6 \mathrm{H}_{2} \mathrm{O}$ and PEG8000, $\mathrm{PgC}_{3}$ and PEG8000, and gel formed from PEG8000-based polyMONCs

9. PXRD spectra of EG@MONC and gels based on PEG8000@MONCs

10. Optical microscopic image of gel made from PEG8000@MONCs

11. SEM/EDS studies of the xerogel

12. The overall information of polyMONCs by using PEG with different molecular weights or different ratios of MONCs/PEG S11

13. Photograph of the sol formed from polyMONCs $\quad \mathrm{S} 12$

14. Shear strain dependent and temperature dependent modulus plot for $\quad \mathrm{S} 12$ the gel samples

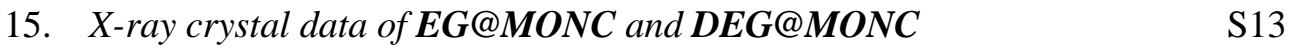

16. References $\quad$ S14 


\section{Materials and methods}

\section{Materials}

All reagents were commercially available and used as supplied without further purification. Solvents were either employed as purchased or dried according to procedures described in the literature. Compound $\mathbf{P g C}_{3}$ and crystal MONC-1 are synthesized according to previous literature. ${ }^{[\mathrm{S} 1]}$

\section{NMR analyses}

All NMR studies were conducted on the Bruker DRX600 instrument at university of Missouri (MU) with a $5 \mathrm{~mm}$ tube. Samples were dissolved in deuterated solvents.

\section{Crystallographic data}

Single-crystal XRD data were collected on a Bruker D8 Venture diffractometer using Mo-K $\alpha$ radiation $(\lambda=0.7179 \AA)$ from a microfocus source. Crystals were cooled to $100 \mathrm{~K}$ during collection. Hemispheres of data were collected out to a resolution of at least $0.80 \AA$ using strategies of scans about the phi and omega axes. High angle data were essentially absent for the crystal of DEG@MONC, so the data set was truncated $0.90 \AA$ during integration. Unit cell determination, data reduction, absorption correction, and scaling were performed using the Bruker Apex3 software suite ${ }^{\mathrm{S} 2}$.

Both crystal structures were solved by an iterative dual space approach as implemented in SHELXT ${ }^{\mathrm{S} 3}$ and refined by full-matrix least squares refinement using SHELXL ${ }^{\mathrm{S} 4}$, implemented via Olex $2^{\mathrm{S} 5}$. All non-hydrogen atoms for the metal ions, $\mathrm{PgC}_{3}$ moieties, and bridging EG/DEG moieties could be located from the difference map and refined anisotropically. For EG@MONC, difference map peaks were observed inside the capsule which were modeled as chelating EG molecules with disordered carbon atoms; these were either refined anisotroipcally at full occupancy (resulting in extremely prolate ellipsoids) or modeled over two positions with the major conformer refined anisotropically. For DEG@MONC, two unique axial ligands could be unambiguously identified as pyridine and nitrate, while for all other ligands only the coordinating atom could be reliably modeled. These ligands are expected to be DEG molecules which are coordinated at one end and disordered at the other end. Due to the low diffraction cutoff for DEG@MONC as well as the fact that it is an exceptionally complicated model, rigid-group restraints were added to the 
atomic displacement parameters for all anisotropic atoms ${ }^{\mathrm{S} 6}$. Additionally, to improve the data to parameters ratio, the disorder model for DEG@MONC was simplified by allowing some disordered atoms to refine anisotropically at full occupancy and removing others. This results in some isolated atoms, atoms with extremely prolate ellipsoids, and some deviating C-C bond distances. The low diffraction cutoff and unomdeled disorder do not affect the most critical aspect of the crystal structure, which is that it proves that hexameric MONCs are crosslinked by DEG into a coordination polymer, and the elemental assignment is strongly supported by the fact that the all parts of the model are consistent with the well-known chemical entities which were used to make the structure.

Hydrogen atoms bonded to carbon atoms were placed in calculated positions and constrained to ride on the carrier atoms in both structures. In EG@MONC hydrogen atoms could be located from the difference map for all protonated phenolic oxygen atoms and most of the EG ligands. For phenolic hydrogen atoms, which are disordered between the two adjacent oxygen atoms in all cases, the coordinates were refined freely and the isotropic thermal parameter was constrained to ride on the nearest oxygen atom. For $-\mathrm{OH}$ groups on EG molecules the $\mathrm{O}-\mathrm{H}$ distances were restrained and the $\mathrm{H}$ atom thermal parameters were constrained to ride on the $\mathrm{O}$ atoms. In both structures, oxygen atoms for which nearby hydrogen atoms could not be located from the difference map were inevitably associated with regions of disordered solvent or interactions with disordered molecules, so these atoms were omitted completely from the model although they were included in the formula.

Both atoms contained regions intermolecular space in which the difference map could not be interpreted, so contributions of disordered matter to the diffraction pattern were masked out using PLATON SQUEEZE ${ }^{\text {S7 }}$. In the case of DEG@MONC this likely includes the tails of coordinating DEG molecules, which are incompletely removed and result in some residual, unmodeled difference map peaks. For EG@MONC two-component twinning was included in the model with the following twin law: $|(-1,-1,0)(0,0,1)(0,0,-1)|$. The fraction corresponding to the minor domain refined to $5.43(8) \%$.

\section{FTIR analyses}

FTIR experiments were conducted on Nicolet Summit FTIR Spectrometer at MU. 


\section{TGA analyses}

TGA were performed on TA instruments ${ }^{T M}$ at MU. Small amount of sample was subjected to heat at constant rate in nitrogen atmosphere. Change in weight percentage was plotted against temperature ranging from room temperature to $800{ }^{\circ} \mathrm{C}$.

\section{SEM analyses}

SEM images were obtained in Quanta 600F Environmental Scanning Electron Microscope at a $\mathrm{HV}=10.00 \mathrm{kV}$. Samples were first dissolved in acetone and then put one drop on a silicon wafer following vacuum drying. Gels were spread on the silicon wafers directly. Before the SEM experiment, samples were sent to sputter coat with platinum. EDS experiments were performed on SEM sample upon Bruker Quantax 200.

\section{TEM and STEM analyses}

TEM and STEM images were obtained in FEI Tecnai F30 Twin 300kV Transmission Electron Microscope. Samples were first dissolved in acetone and then put one drop on a carbon film.

\section{PXRD analyses}

PXRD data were collected on a PANalytical X'Pert PRO X-ray diffractometer operating at $40 \mathrm{kV} / 40 \mathrm{~mA}$ using the $\mathrm{Cu} \mathrm{K}_{\alpha 1}(\lambda=1.540598 \AA)$ and $\mathrm{K}_{\alpha 2}(\lambda=1.544426$ A).

\section{Rheological Test}

The rheological behavior of the gels was characterized with a DHR-2 rheometer (TA Instruments). A disk-shaped sample with a diameter of $25.0 \mathrm{~mm}$ and a thickness of $1.2 \mathrm{~mm}$ was fixed between two metal plates using super glue. Frequency sweeps were performed from 0 to $100 \mathrm{rad} / \mathrm{s}$ at $2 \%$ strain. Strain-sweep and temperature-sweep rheology experiments were conducted on Kinexus Pro (Malvern Panalytical Inc., Westborough, MA). Strain-sweep was performed with strain from $0.1 \%$ to $800 \%$, with a frequency of $1.6 \mathrm{~Hz}$, at $25{ }^{\circ} \mathrm{C}$. Temperature-sweep was performed from $25-90{ }^{\circ} \mathrm{C}$, with a frequency of $1.6 \mathrm{~Hz}$ and a strain of $10 \%$. To minimize the solvent evaporation on such small-volume samples, the measurement was conducted 
immediately after the sample was loaded onto the washer. In temperature-sweep, the loaded gel samples were immersed in mineral oil to reduce solvent evaporation.

\section{Small angle $X$-ray scattering analyses}

Small angle X-ray scattering analyses was characterized with Xenocs SAXS equipment. The experiment was conducted in $1200 \mathrm{~s}$, under a power of $50 \mathrm{kV}, 0.6 \mathrm{~mA}$, using a PILATUS $100 \mathrm{~K}$ detector and the wavelength was 0.15148 .

\section{Synthesis and Crystallization of EG@MONC.}

$0.030 \mathrm{~g}$ of $\mathbf{P g C} 3$ and $0.0427 \mathrm{~g}$ of $\mathrm{Mg}\left(\mathrm{NO}_{3}\right)_{2} \bullet 6 \mathrm{H}_{2} \mathrm{O}$ was dissolved in $1 \mathrm{~mL} \mathrm{CH}_{3} \mathrm{CN}$ and $1 \mathrm{~mL} \mathbf{E G}$, to which $50 \mu \mathrm{L}$ of pyridine was added after dissolution. The $\mathbf{P g C}_{\mathbf{3}}$ and magnesium solutions were mixed in a $4 \mathrm{~mL}$ vial, and was sonicated for $5 \mathrm{~min}$. After heating at $100{ }^{\circ} \mathrm{C}$ overnight, green crystals were formed and collected for single crystal X-ray analysis. The yield is $49 \%$.

\section{Synthesis and Crystallization of DEG@MONC.}

$0.030 \mathrm{~g}$ of $\mathbf{P g C}_{3}$ and $0.0427 \mathrm{~g}$ of $\mathrm{Mg}\left(\mathrm{NO}_{3}\right)_{2} \cdot 6 \mathrm{H}_{2} \mathrm{O}$ were dissolved in $1 \mathrm{~mL}$ $\mathrm{CH}_{3} \mathrm{CN}$ and $1 \mathrm{~mL}$ DEG, to which $50 \mu \mathrm{L}$ of pyridine was added after dissolution. The solution was sonicated for $5 \mathrm{~min}$. After heating at $100{ }^{\circ} \mathrm{C}$ overnight, green crystals were formed and collected for single crystal X-ray analysis. The yield is $41 \%$.

\section{Synthesis of polyMONCs.}

$0.120 \mathrm{~g}$ of $\mathbf{P g C} \mathbf{C}_{3}, 0.171 \mathrm{~g}$ of $\mathrm{Mg}\left(\mathrm{NO}_{3}\right)_{2} \bullet 6 \mathrm{H}_{2} \mathrm{O}$ and PEG (the molecular weight and the quantity depend on the circumstance we designed) were dissolved in $5 \mathrm{~mL}$ $\mathrm{CH}_{3} \mathrm{CN}$, to which $50 \mu \mathrm{L}$ of pyridine was added after dissolution. The solution was sonicated for $5 \mathrm{~min}$ and then stirring at $120{ }^{\circ} \mathrm{C}$ overnight. The product was separated by centrifuging from the reaction mixture. 
2. The crystal structure of MONC-1

(a)

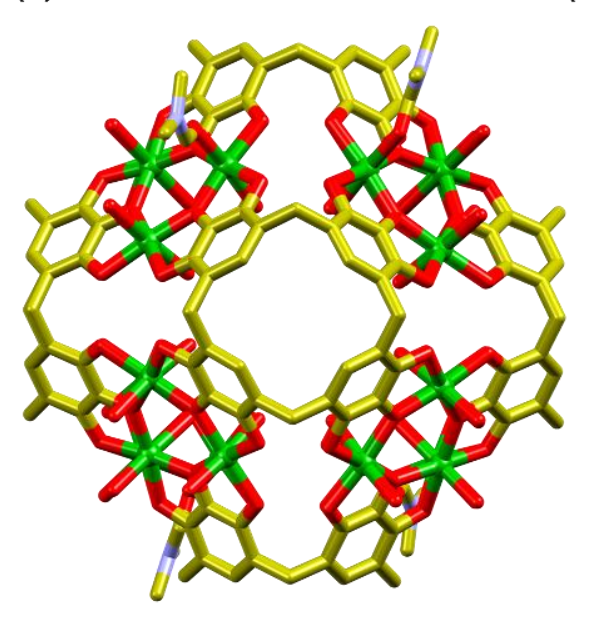

(b)

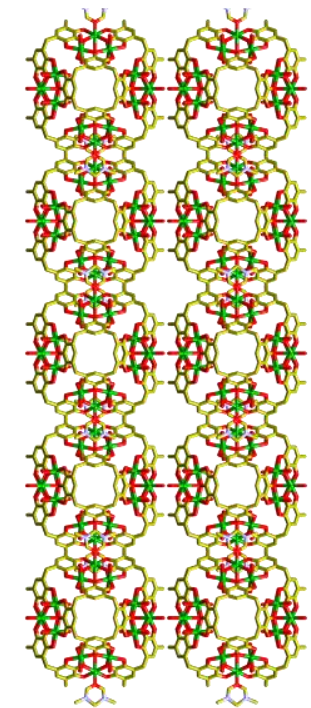

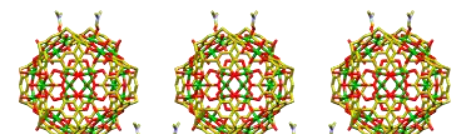

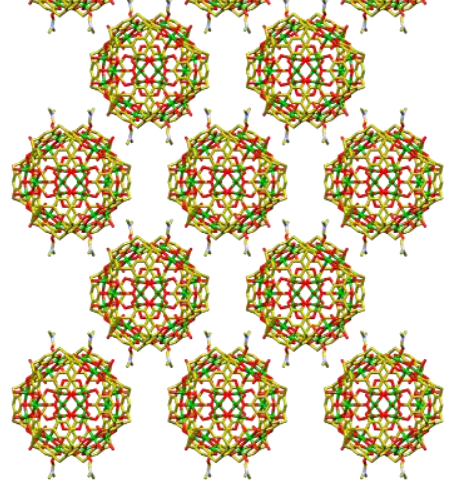

Figure S1. (a) The crystal structure of MONC-1. (b) Extended view of MONC-1. Color codes: magnesium atoms are green; carbon atoms are yellow; oxygen atoms are red. Hydrogen atoms, and propyl-tails of $\mathbf{P g C}_{\mathbf{3}}$ were removed for clarity. ${ }^{\mathrm{S} 1}$

3. TEM and STEM images of PEG400@MONCs and PEG8000@MONCs
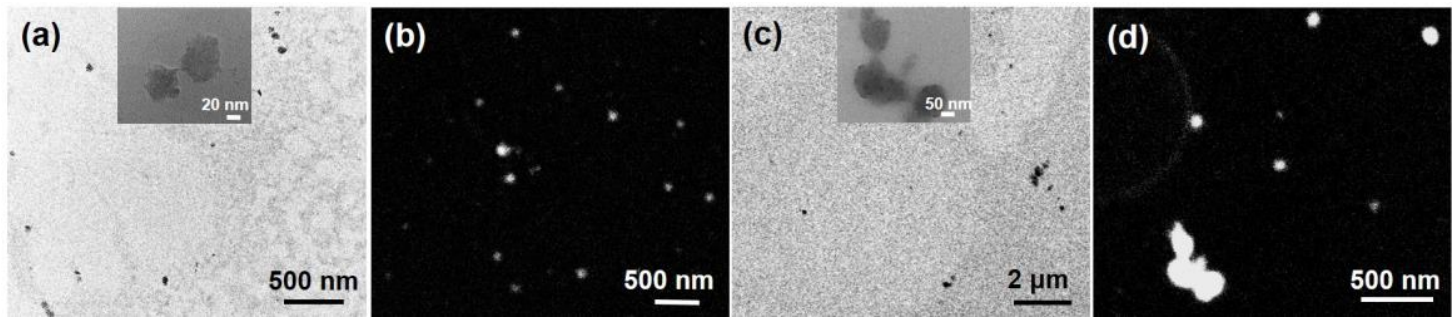

Figure S2. (a) TEM images of PEG400@MONCs; (b) STEM image of PEG400@MONCs; (c) TEM images of PEG8000@MONCs; (d) STEM image of PEG8000@MONCs. 
4. ${ }^{1}$ H NMR spectra of MONC-1,EG@MONC,PEG400@MONCs and PEG8000@MONCs

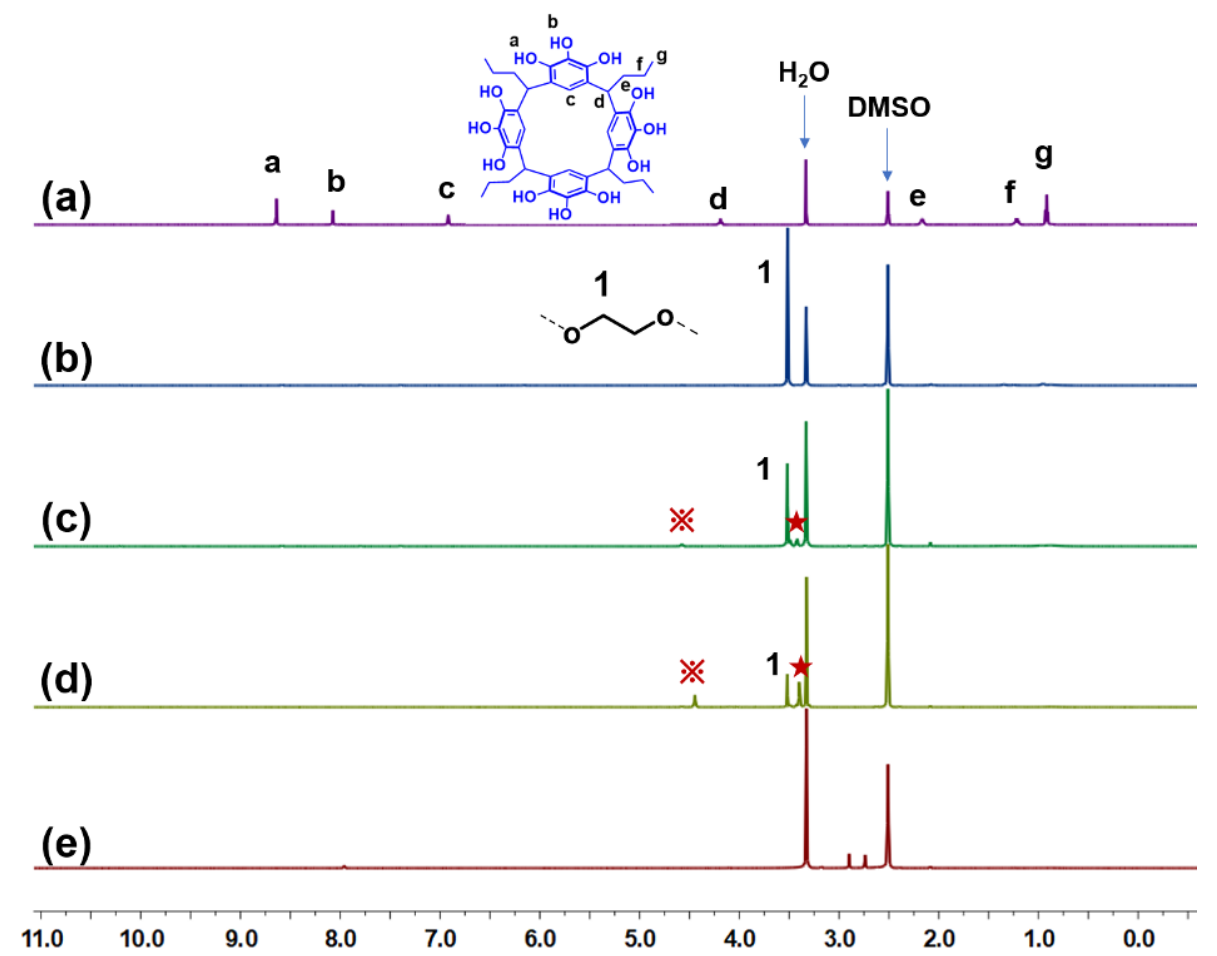

Figure S3. Partial ${ }^{1} \mathrm{H}$ NMR spectra (600 MHz, DMSO- $d_{6}$, room temperature) of (a) $\mathbf{P g C}_{3}$; (b) PEG8000@MONCs; (c) PEG400@MONCs; (d) EG@MONC; (e) MONC-1. ( $※$ and $\star$ in Figure S3c and S3d represented the coordinated protons of $\mathrm{H}_{1}$.)

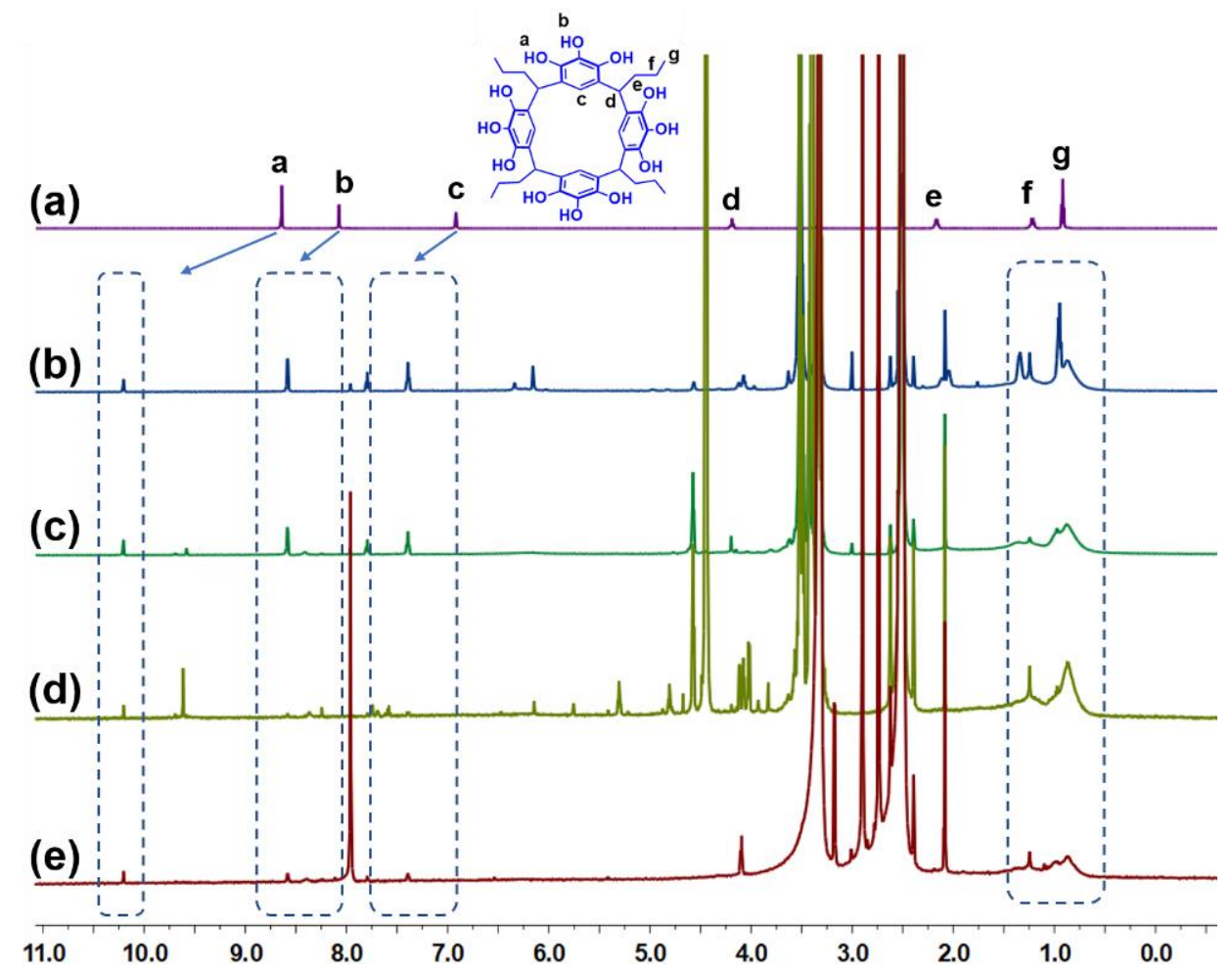

Figure S4. Enlarged ${ }^{1} \mathrm{H}$ NMR spectra (600 MHz, DMSO- $d_{6}$, room temperature) of Figure S3: (a) PgC ; (b) PEG8000@MONCs; (c) PEG400@MONCs; (d) EG@MONC; (e) MONC-1. 
As shown in Figure $\mathrm{S} 3$, the protons $\mathrm{H}_{1}$ belong to the methylene part on EG or PEG can be found in the ${ }^{1} \mathrm{H}$ NMR spectra of EG@MONC, PEG400@MONCs and PEG8000@MONCs, indicating that the PEG chains were indeed contained in these materials. From the enlarged ${ }^{1} \mathrm{H}$ NMR spectra (Figure S4), protons signals assigned to the coordinated $\mathbf{P g C}_{\mathbf{3}}$ could be detected in the spectra of non-crosslinked MONCs (MONC-d, Figure S1), EG@MONC, PEG400@MONCs and PEG8000@MONCs. Moreover, protons signals on PEG400@MONCs and PEG8000@MONCs possess the similar chemical shift and broaden effect compared with that of EG@MONC, illustrating the formation of crosslinked MONCs in all of these materials.

5. FTIR spectra of EG@MONC,PEG400@MONCs and PEG8000@MONCs

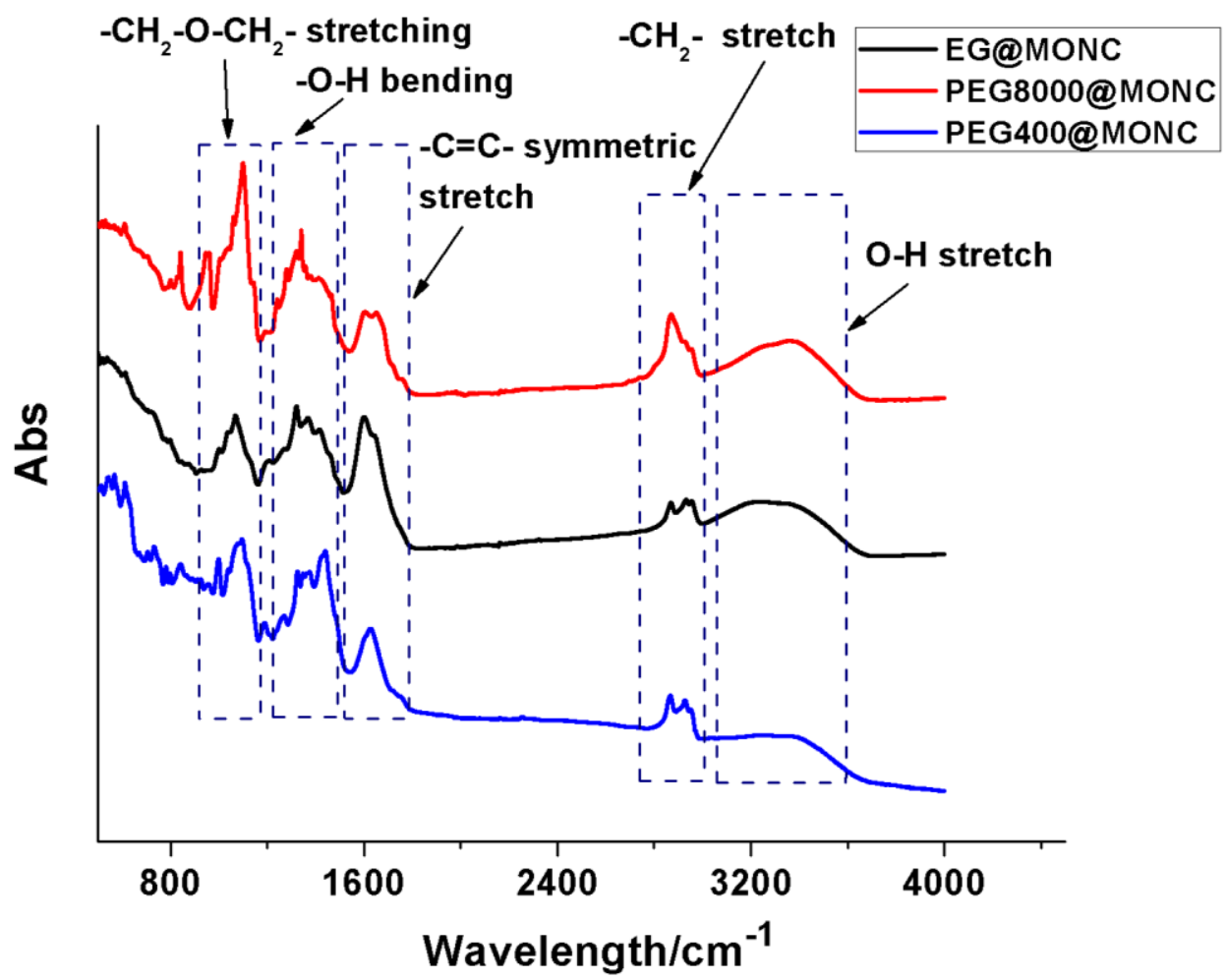

Figure S5. FTIR spectra of EG@MONC, PEG400@MONCs and PEG8000@MONCs.

The overall FTIR spectra of EG@MONC, PEG400@MONCs and PEG8000@MONCs were similar and the absorption peaks of the stretching vibration bands $\left(2858-2950 \mathrm{~cm}^{-1}\right.$, $1010-1110 \mathrm{~cm}^{-1}, 3280-3380 \mathrm{~cm}^{-1}, 1620-1650 \mathrm{~cm}^{-1}$ ) derived from $-\mathrm{CH}_{2^{-}},-\mathrm{CH}_{2}-\mathrm{O}-\mathrm{CH}_{2-},-\mathrm{OH}$ and benzene ring on PEG and $\mathbf{P g C} \mathbf{C}_{3}$ were easily observed in FTIR spectra. 
6. TGA spectra of MONC-1,EG@MONC,PEG400@MONCs and PEG8000@MONCs

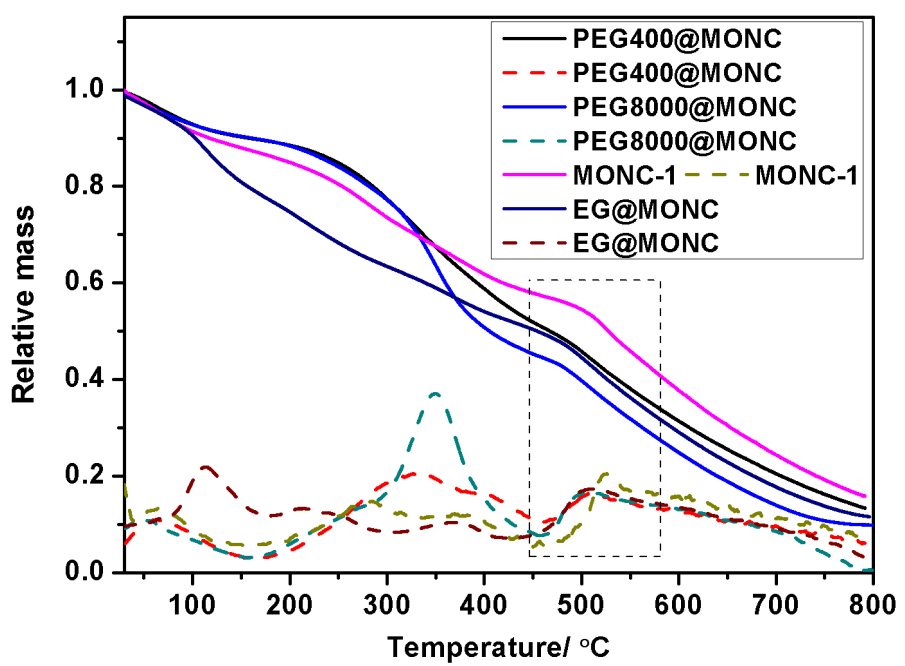

Figure S6. TGA spectra of MONC-1, EG@MONC, PEG400@MONCs and PEG8000@MONCs. The dash lines represent the derivative value of solid line.

The thermal stabilities of MONC-1, EG@MONC, and polyMONCs based on PEG400 and PEG8000 were measured by TGA (Figure S6). The PEG chains decomposed at about $350^{\circ} \mathrm{C}$, and the MONCs decomposed at around $500{ }^{\circ} \mathrm{C}$ to EG@MONC and polyMONCs and $530{ }^{\circ} \mathrm{C}$ to MONC-1, resulting from the high thermal stability.

\section{Optical microphotographs of MONC-1 and EG@MONC}

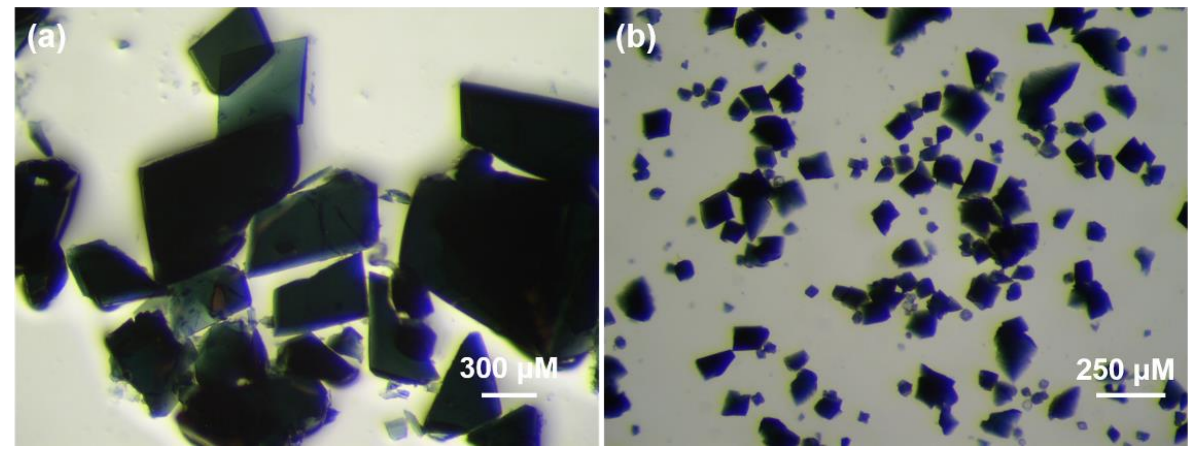

Figure S7. Optical microphotographs of (a) MONC-1 and (b) EG@MONC.

Optical micrographs of MONC-1, and EG@MONC illustrated that the size of the crystals could be reduced after the addition of EG (Figure S7). 
8. Photographs of the solution formed from $\mathrm{Mg}\left(\mathrm{NO}_{3}\right)_{2} \cdot 6 \mathrm{H}_{2} \mathrm{O}$ and $\mathrm{PEG} 8000, \mathrm{PgC}_{3}$ and PEG8000, and gel formed from PEG8000-based polyMONCs

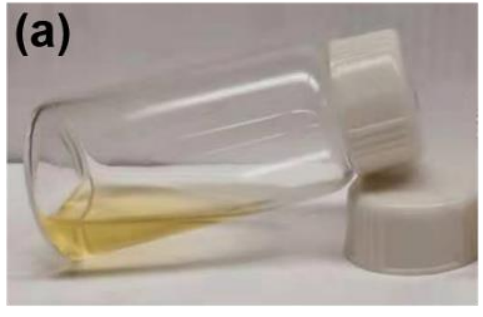

$\mathrm{Mg}\left(\mathrm{NO}_{3}\right)_{2} \cdot 6 \mathrm{H}_{2} \mathrm{O}$ and PEG8000

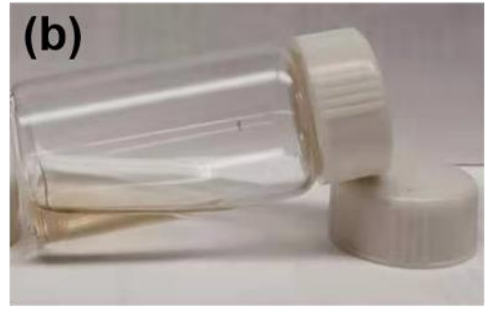

$\mathrm{PgC}_{3}$ and PEG8000 (c)

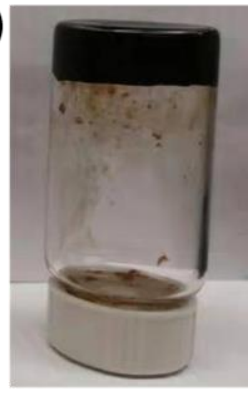

Gel

Figure S8. Photographs of the solution formed from (a) $\mathrm{Mg}\left(\mathrm{NO}_{3}\right)_{2} \cdot 6 \mathrm{H}_{2} \mathrm{O}$ and PEG8000 $\left(\mathrm{Mg}\left(\mathrm{NO}_{3}\right)_{2} \cdot 6 \mathrm{H}_{2} \mathrm{O} / \mathrm{PEG} 8000=4: 1\right),(\mathrm{b}) \mathbf{P g C}_{3}$ and PEG8000 $\left(\mathbf{P g C}_{3} / \mathrm{PEG} 8000=1: 1\right)$, and (c) gel formed from polyMONCs based on PEG8000@MONCs (MONCs/PEG8000 = 1:6, 15 $\mathrm{wt} \%$ in acetonitrile).

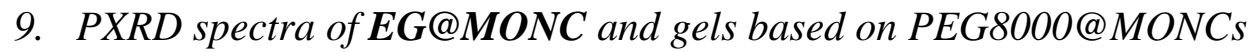

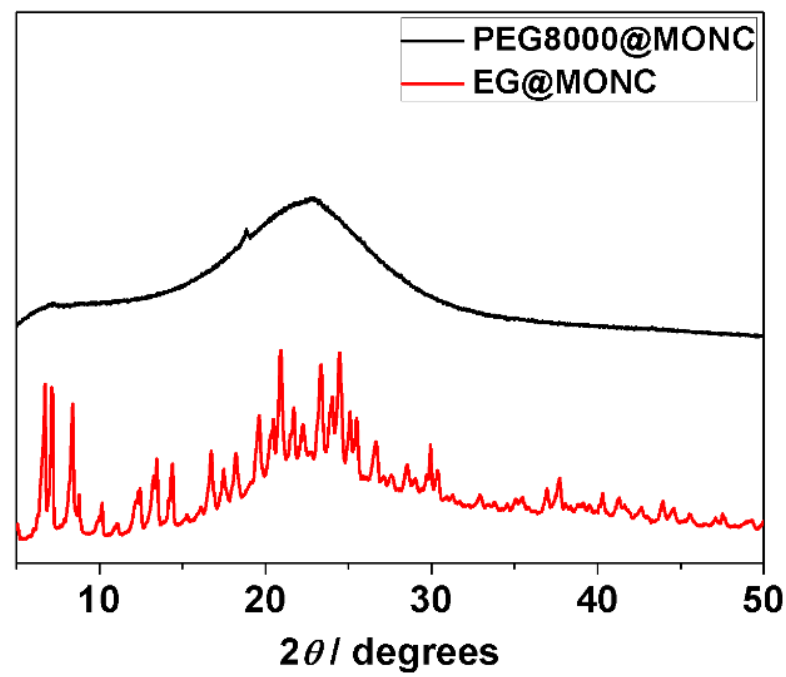

Figure S9. PXRD spectra of EG@MONCs and gels based on PEG8000@MONCs. 
10. Optical microscopic image of gel made from PEG8000@MONCs

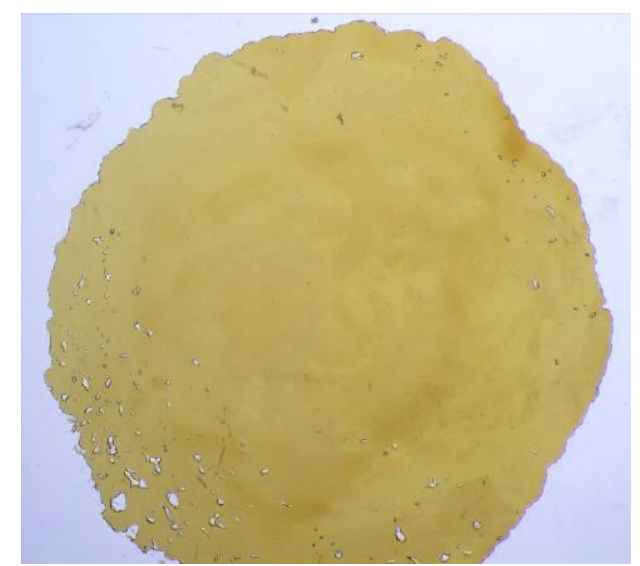

Figure S10. Optical microscopic image of gel made from PEG8000@MONCs $(\mathrm{MONCs} / \mathrm{PEG} 8000=1: 6,15 \mathrm{wt} \%$ in acetonitrile), illuminated by transmission at $\sim 7.5 \mathrm{x}$ magnification.

\section{SEM/EDS studies of the xerogel}

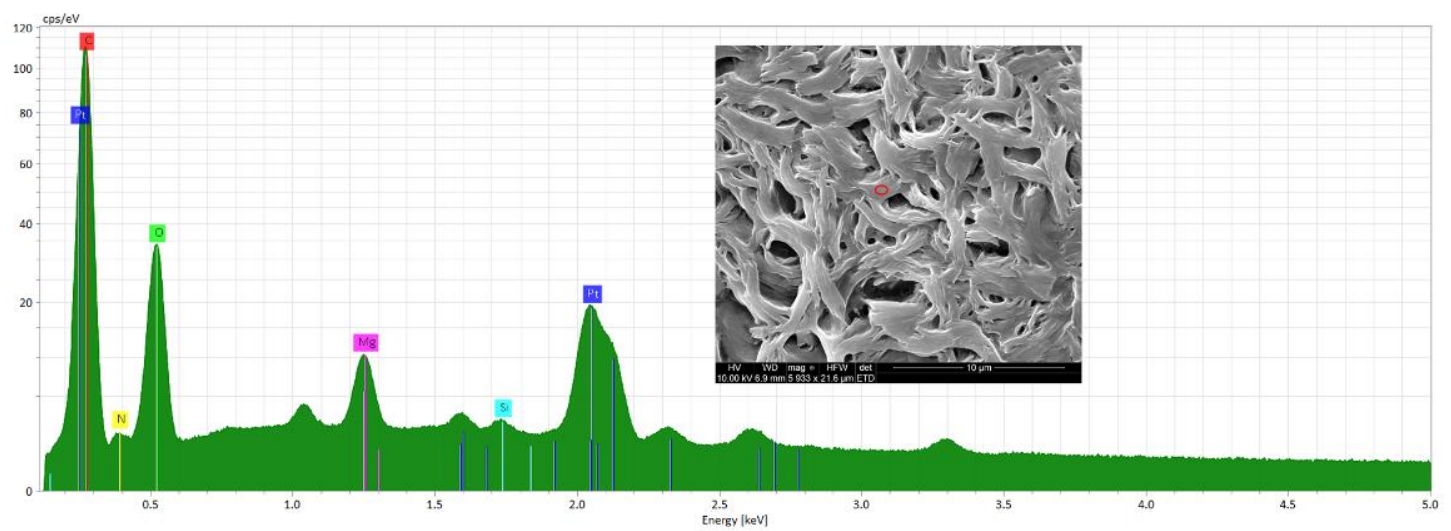

Figure S11. SEM/EDS studies of the xerogel. The red part of the sample was selected for EDS measurement.

12. The overall information of polyMONCs by using PEG with different molecular weights or different ratios of MONCs/PEG

\begin{tabular}{|c|c|c|c|c|c|}
\hline & $\begin{array}{c}\text { PEG200 } \\
0\end{array}$ & $\begin{array}{c}\text { PEG400 } \\
0\end{array}$ & $\begin{array}{c}\text { PEG600 } \\
0\end{array}$ & $\begin{array}{c}\text { PEG800 } \\
0\end{array}$ & $\begin{array}{c}\text { PEG100 } \\
00\end{array}$ \\
\hline $1: 4$ & $/$ & $/$ & $/$ & gel-2 & $/$ \\
$1: 6$ & sol & sol & gel-1 & gel-3 & gel-4 \\
$1: 8$ & $/$ & $/$ & $/$ & sol & $/$ \\
\hline
\end{tabular}

Table. S1 The overall information of polyMONCs by using PEG with different molecular weights or different ratios of MONCs/PEG (15 wt\% in acetonitrile). 
13. Photograph of the sol formed from polyMONCs

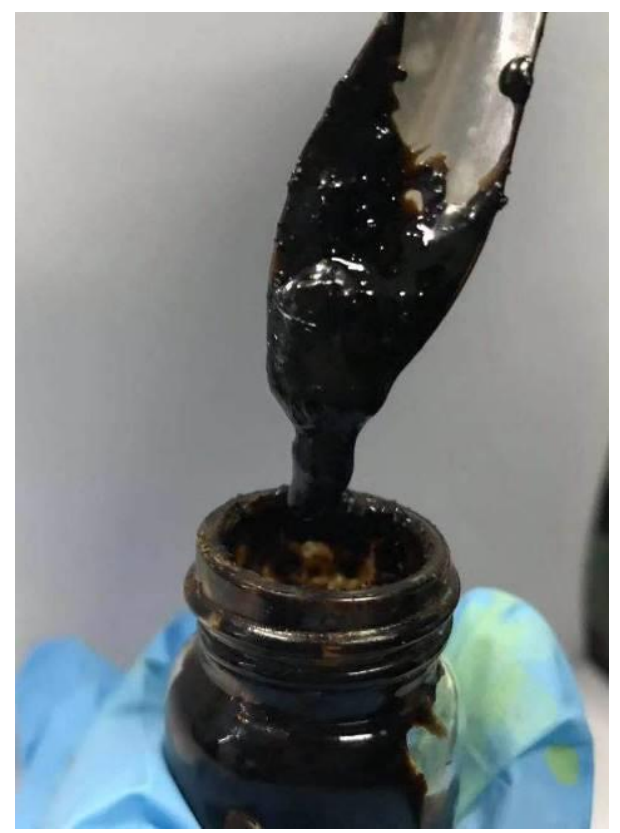

Figure S12. Photograph of the sol formed from polyMONCs.

14. Shear strain dependent and temperature dependent modulus plot for the gel samples
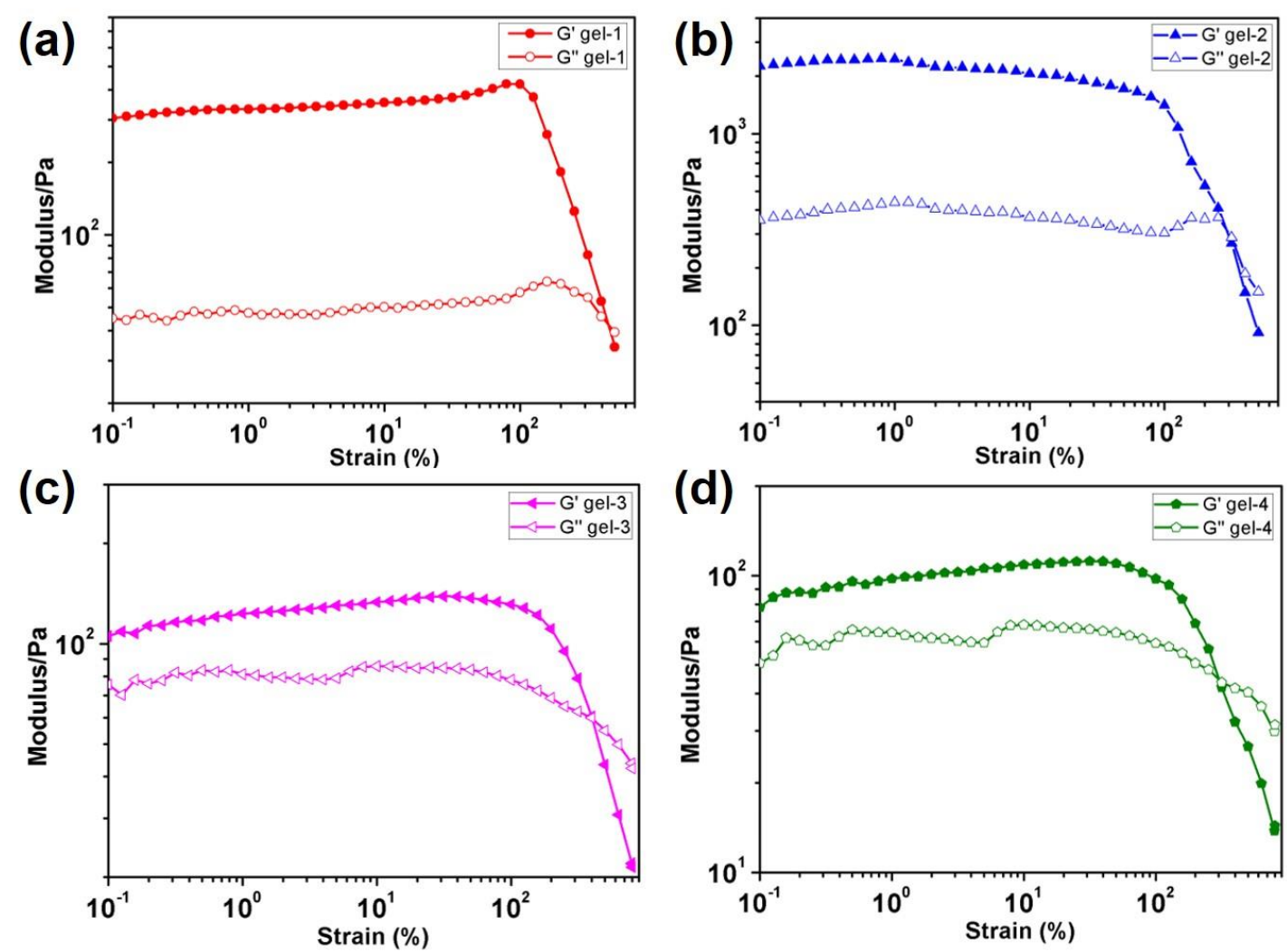

Figure S13. The strain-sweep rheology data for (a) gel-1, (b) gel-2, (c) gel-3 and (d) gel-4. 


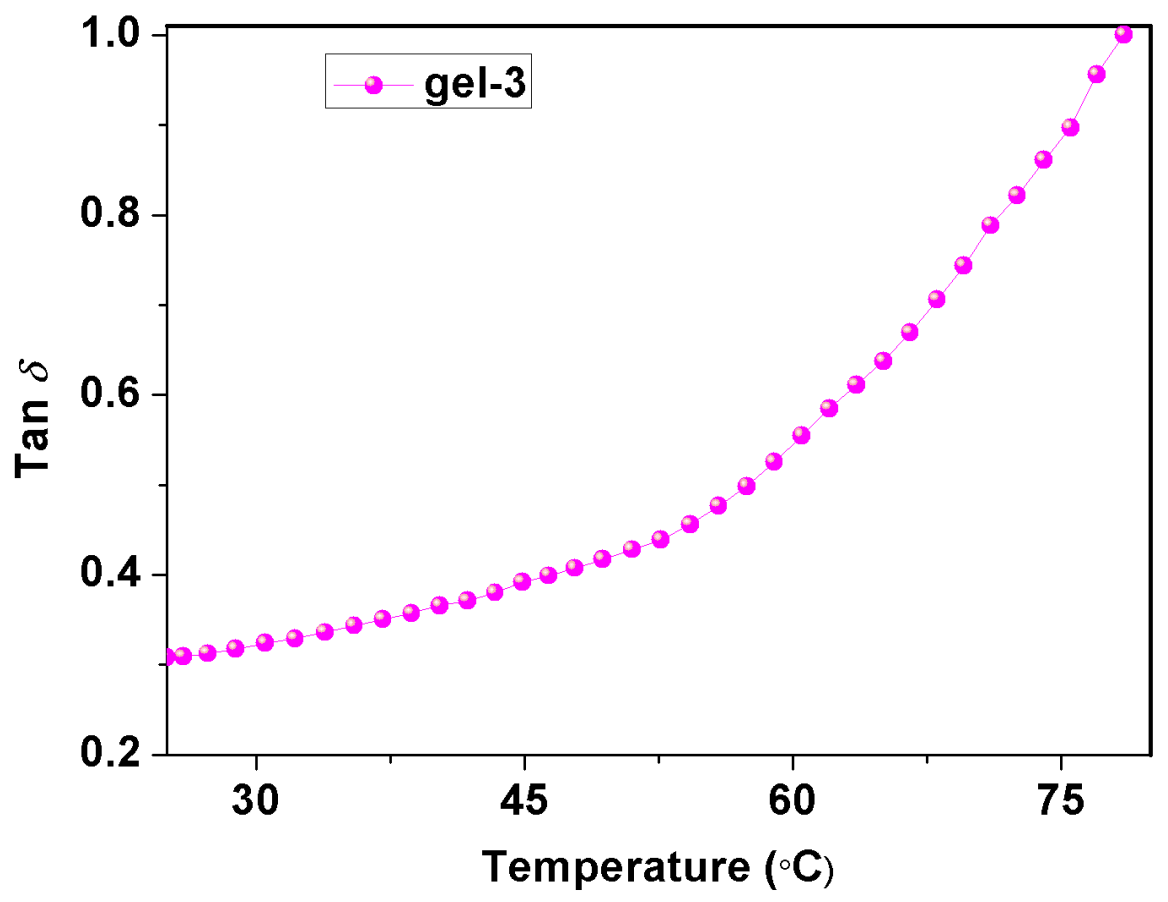

Figure S14. Temperature-sweep rheology data of gel-3. The crossover point of G' and $\mathrm{G}^{\prime \prime}$ ( $\operatorname{Tan} \delta=1.0$ ) shows the transition from gel to liquid.

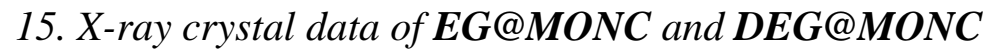

Formula

Crystallization Solvent

Collection Temperature

Sum Formula

$\mathrm{Mr}$

Crystal Size [mm]

Crystal System

Space Group

$a[\AA]$

$b[\AA]$

$c[\AA]$

$\alpha\left[^{\circ}\right]$

$\beta\left[^{\circ}\right]$

$\gamma\left[^{\circ}\right]$

$V\left[\AA^{3}\right]$

$Z$

Dcalcd $\left[\mathrm{g} \mathrm{cm}^{-3}\right]$

\section{EG@MONC}

acetonitrile/ethylene glycol acetonitrile/diethylene glycol

$100 \mathrm{~K}$

$100 \mathrm{~K}$

$\mathrm{C}_{300} \mathrm{H}_{420} \mathrm{Mg}_{24} \mathrm{O}_{138}$

6817.77

$\mathrm{C}_{265} \mathrm{H}_{296} \mathrm{Mg}_{24} \mathrm{~N}_{2} \mathrm{O}_{129}$

6156.46

$0.24 \times 0.15 \times 0.05$

trigonal

R-3

34.417(3)

34.417(3)

26.544(2)

90

90

120

27230(5)

3

1.247
$0.25 \times 0.14 \times 0.1$

monoclinic

$\mathrm{P} 2{ }_{1} / \mathrm{c}$

$37.025(5)$

36.916(5)

26.530(4)

90

94.801(4)

90

36134(9)

4

1.132 


$\begin{array}{ccc}\mu\left[\mathrm{mm}^{-1}\right] & 0.134 & 0.126 \\ \mathrm{~F}(000) & 10836.0 & 12880.0 \\ 2 \theta \text { range }\left[^{\circ}\right] & 4.734-52.846 & 4.33-46.694 \\ \text { Reflections collected } & 157796 & 405391 \\ \text { Independent reflections, Rint } & 12438,0.1174 & 52201,0.0841 \\ \text { Data /restraints /parameters } & 12438 / 3 / 758 & 52201 / 5148 / 3781 \\ \text { Final } R \text { 1 values }(I>2 \sigma(I)) & 0.0810 & 0.0890 \\ \text { Final } R \text { ( values (all data) } & 0.1325 & 0.1382 \\ \text { Final } w R\left(F_{2}\right) \text { values (all data) } & 0.2488 & 0.2987 \\ \text { Goodness-of-fit on } F^{2} & 1.023 & 1.027 \\ \text { Largest difference peak and hole }\left[\mathrm{e} . \mathrm{A}^{-3}\right] & 0.76 /-0.46 & 1.50 /-0.87 \\ \text { CCDC } & 1977717 & 1977732\end{array}$

\section{References}

S1. Zhang C.; Patil S. R.; Li T.; Barnes L. C.; Teat J. S.; Atwood L. J. Preparation of Magnesium-Seamed C-Alkylpyrogallol[4]arene Nanocapsules with Varying Chain Lengths. Chem. Eur. J. 2017, 23, 8520-8524.

S2. Apex3, AXScale, and SAINT, version 2017.3-0, Bruker AXS, Inc., Madison, WI, 2017.

S3 Sheldrick, G. M. SHELXT - Integrated space-gropu and crystal-structure determination. Acta Cryst. Sect. A: Found. Adv. 2015, 71, 3-8.

S4 Sheldrick, G. M. Crystal structure refinement with SHELXL. Acta Cryst. Sect. C. Struct. Chem. 2015, 71, 3-8.

S5 Dolomanov, O.V.; Bourhis, L.J.; Gildea, R.J.; Howard, J.A.K.; Puschmann, H. OLEX2: A complete structure solution, refinement, and analysis program. J. Appl. Cryst. 2009, 42, 339-341.

S6 Thorn, A.; Dittrich, B.; Sheldrick, G. M. Enhanced rigid-bond restraints. Acta Cryst. Sect. A. Found. Adv. 2012, 68, 448-451.

S7. Spek, A. L. PLATON SQUEEZE: A tool for the calculation of the disordered solvent contribution to the calculated structure factors. Acta Cryst. Sect. C. Struct. Chem. 2015, 71, 9-18. 